\title{
Produção de mudas de laranjeira Pêra por meio do método de interenxertia
}

\author{
Production of nursery trees of Pera sweet orange by the interstock method \\ Denilson de Oliveira Guilherme ${ }^{\mathrm{I}^{*}}$ Cláudia Sales Marinho $^{\mathrm{I}}$ Marlon Altoé Biazatt ${ }^{\mathrm{I}}$ \\ Graziella Siqueira Campos ${ }^{\mathrm{I}}$ Cintia Aparecida Bremenkamp ${ }^{\mathrm{I}}$
}

\section{- NOTA -}

\section{RESUMO}

A interenxertia é uma prática que é usada, quando se deseja unir duas plantas que, sabidamente, são incompatíveis, ou quando se pretende diminuir o vigor da cultivar copa. Neste trabalho, avaliou-se uma metodologia alternativa para a produção de mudas de laranjeira Pêra interenxertadas, usando a subenxertia como técnica auxiliar na formação de um interenxerto de limoeiro Cravo. Foram avaliados os seguintes tratamentos: T1 - portaenxerto trifoliata Flying Dragon (FD) e interenxerto de laranjeira Bahia; T2 - porta-enxerto Citrumeleiro Swingle (CS) e interenxerto de laranjeira Bahia; T3 - porta-enxerto limoeiro Cravo (LC); T4 - porta-enxerto FD e interenxerto de LC; T5 - Porta-enxerto CS e interenxerto de LC. As mudas produzidas no tratamento 4 (Pêra / LC / FD) se aproximaram em vigor das mudas produzidas sem filtro (Pêra / LC). A produção de mudas de laranjeira Pêra interenxertadas pelo sistema tradicional, tendo como porta-enxerto CS ou FD e interenxerto de laranjeira Bahia, não foi possível, em virtude da baixa viabilidade e vigor das brotações.

Palavras-chave: Poncirus trifoliata, Flying Dragon, Citrumeleiro Swingle, subenxertia, incompatibilidade de enxertia.

\section{ABSTRACT}

The interstock is a practice used when to merge two plants that are known to be incompatible or when it is intended to low vigor of the scion. In this study we evaluated an alternative method for the production of Pêra sweet orange nursery trees interstocked, using the technique as inarching assist in forming a interstock of Rangpur Lime (RL). The treatments were: $T 1$ - rootstock trifoliate Flying Dragon (FD) and interstock of sweet orange Bahia, T2 - rootstock Citrumelo Swingle (CS) and interstock of sweet orange Bahia, T3 - rootstock Rangpur Lime (RL); T4 - rootstock FD and interstock RL; T5 - rootstock CS and interstock RL. The sweet orange nursery trees produced in treatment 4 (Pêra / RL / $F D)$ had vigor nearest sweet orange nursery trees produced with interstock (Pêra / RL). The production Pera sweet orange nursery trees interstocked for traditional system, with the rootstock CS or FD and interstock sweet orange Bahia was not possible because of the low viability and vigor of the shoots.

Key words: Poncirus trifoliata, Flying Dragon, Citrumelo Swingle, grafting incompatibility, inarching.

A laranjeira Pêra é uma das cultivares de laranja doce mais plantadas no Brasil, devido a sua versatilidade de uso tanto na indústria quanto para o consumo in natura. O limoeiro Cravo é ainda o porta-enxerto mais utilizado em extensas áreas de produção e sua diversificação tem sido recomendada, principalmente após o surgimento do declínio e da morte súbita (POMPEU JUNIOR \& BLUMER, 2008).

A diversificação de porta-enxertos para a laranjeira Pêra tem sido mais restrita quando relacionada às demais cultivares de laranjeiras, por sua reportada incompatibilidade com o Poncirus trifoliata e seus híbridos, como o citrumeleiro Swingle, além de outros porta-enxertos, como o limoeiro Volkameriano (DONADIO, 1999).

A interenxertia é uma prática que é usada, quando se deseja unir duas plantas que, sabidamente, são incompatíveis, ou quando se pretende diminuir o vigor da cultivar copa. Essa técnica consiste em interpor um fragmento de uma planta entre o enxerto e o porta-enxerto. Assim, uma planta interenxertada apresenta três partes geneticamente diferentes (porta-enxerto, interenxerto e enxerto) e dois locais de enxertia (FACHINELLO et al., 2005). No caso

'Universidade Estadual do Norte Fluminense Darcy Ribeiro (UENF), 28013-602, Campos dos Goytacazes, RJ, Brasil. E-mail: doliveiraguilherme@yahoo.com.br.*Autor para correspondência. 
da laranjeira Pêra, sua incompatibilidade com os trifoliatas ou seus híbridos pode ser contornada com a interenxertia de um fragmento de outra laranjeira doce, entre os tecidos desses porta-enxertos e os tecidos da Pêra. O uso do citrumeleiro para a Pêra pode ser desejável por sua resistência à gomose, nematoides e morte súbita. O Flying dragon pode ser desejável como porta-enxerto para a Pêra, por ser um porta-enxerto nanicante e permitir maior adensamento de plantio. Nos casos citados, as mudas de Pêra sobre esses porta-enxertos precisariam ser produzidas por interenxertia. Entretanto, o método convencional de produção de mudas de citros com interenxerto leva a alto índice de descarte de plantas e maior tempo de produção da muda, encarecendo o seu custo de produção (GIRARDI \& MOURÃO FILHO, 2006). Sendo assim, inovações técnicas na produção de mudas interenxertadas devem ser investigadas para maior eficiência na produção.

O objetivo deste trabalho foi avaliar a subenxertia como técnica auxiliar para a produção de mudas de laranjeira Pêra interenxertadas, com limoeiro Cravo, em comparação ao método convencional de produção de mudas interenxertadas com uma laranjeira doce.

O experimento foi instalado em viveiro protegido com telado antiafideo, na Universidade Estadual do Norte Fluminense Darcy Ribeiro, em Campos dos Goytacazes - RJ. Utilizou-se delineamento em blocos casualizados, com cinco tratamentos, quatro repetições e dez plantas por parcela. Foram utilizados os porta-enxertos Poncirus trifoliata var. monstrosa Flying Dragon (Poncirus trifoliata var. monstrosa T. Ito) (FD), limoeiro Cravo (Citrus limonia Osbeck) (LC) e o citrumeleiro Swingle (Citrus paradisi X Poncirus trifoliata) (CS). Os cinco tratamentos utilizados na formação das mudas de laranjeira Pêra, podem ser observados na tabela 1 .

Os porta-enxertos FD, LC e CS foram semeados em agosto de 2011 em tubetes de $50 \mathrm{~cm} 3$, preenchidos com substrato comercial Basaplant ${ }^{\circledR}$
Hortaliças BX. Ao substrato, foram adicionados, adubo de liberação lenta Osmocote ${ }^{\circledR}$ (14-14-14), superfosfato simples e calcário nas concentrações de 3,0 g L$^{-1}$; 5,0 $\mathrm{g} \mathrm{L}^{-1}$ e 13,0 $\mathrm{g} \mathrm{L}^{-1}$, respectivamente. Sessenta dias após a semeadura, esses porta-enxertos foram transplantados para citropotes de 3,8 L, preenchidos com substrato comercial Basaplant ${ }^{\circledR}$ Hortaliças BX. Ao substrato, foram adicionados, adubo de liberação lenta Osmocote ${ }^{\circledR}$ (17-07-12), superfosfato simples e calcário nas concentrações de 3,0 $\mathrm{g} \mathrm{L}^{-1}$; 5,0 $\mathrm{g} \mathrm{L}^{-1}$ e 13,0 g L ${ }^{-1}$, respectivamente.

Uma nova semeadura dos porta-enxertos FD e CS foi realizada em setembro de 2011 para que estas mudas fossem utilizadas como subenxertos nos tratamentos 4 e 5 . Para isso, os mesmos tipos de recipientes e substratos da primeira semeadura foram utilizados. Essas plantas (FD e CS) foram transplantados para os citropotes, nos quais já vinham sendo cultivadas mudas de LC. Ao lado de cada muda de LC, foi aberta uma cova (volume de $50 \mathrm{~cm}^{3}$ ) para o transplantio do FD e CS. Aos 20 dias após o transplantio do LC, efetuou-se um corte em “T” invertido no caule do LC e neste foi introduzido o subenxerto, que foi cortado em bisel a $15 \mathrm{~cm}$ do colo e introduzido sob a casca do LC. Os subenxertos (FD ou CS) foram protegidos com parafilm e tutorados com hastes de bambu.

Quando o LC, FDeCS, semeados em agosto, atingiram diâmetro de caule (a $10 \mathrm{~cm}$ do colo) entre 5 a $10 \mathrm{~mm}$, foram enxertadas borbulhas das laranjeiras Bahia nos tratamentos 1 e 2 e Pêra nos tratamentos 3, 4 e 5. As mudas de FD e CS enxertadas com borbulhas da laranjeira Bahia seriam posteriormente enxertadas com borbulhas da laranjeira Pêra. Nas mudas provenientes do tratamento 4 e 5, foi realizado anelamento no caule do LC a $5 \mathrm{~cm}$ abaixo da região da subenxertia. O desmame das mudas nestes tratamentos (corte do sistema radicular do LC) foi realizado 60 dias após a enxertia (DAE) das borbulhas de laranjeira Pêra. Um esquema ilustrativo da composição dos tratamentos $4 \mathrm{e}$ 5 é apresentado na figura 1.

Tabela 1 - Tratamentos propostos para formação de mudas de laranjeira Pera com interenxerto de Limoeiro Cravo (LC) ou de laranjeira Bahia.

\begin{tabular}{|c|c|c|c|c|}
\hline Tratamento & Porta-Enxerto & Interenxerto & Enxerto & Método de enxertia \\
\hline 1 & Flying Dragon & Laranjeira Bahia & Laranjeira Pêra & ${ }^{(1)}$ Borbulhia $+{ }^{(2)}$ Borbulhia \\
\hline 2 & Citrumeleiro Swingle & Laranjeira Bahia & Laranjeira Pêra & ${ }^{(1)}$ Borbulhia $+{ }^{(2)}$ Borbulhia \\
\hline 3 & Limoeiro Cravo & - & Laranjeira Pêra & ${ }^{(1)}$ Borbulhia \\
\hline 4 & Flying Dragon & Limoeiro Cravo & Laranjeira Pêra & ${ }^{(3)}$ Subenxertia $+{ }^{(2)}$ Borbulhia \\
\hline 5 & Citrumeleiro Swingle & Limoeiro Cravo & Laranjeira Pêra & ${ }^{(3)}$ Subenxertia $+{ }^{(2)}$ Borbulhia \\
\hline
\end{tabular}

${ }^{(1)}$ No porta-enxerto; ${ }^{(2)}$ no interenxerto; ${ }^{(3)}$ sob casca do LC. 


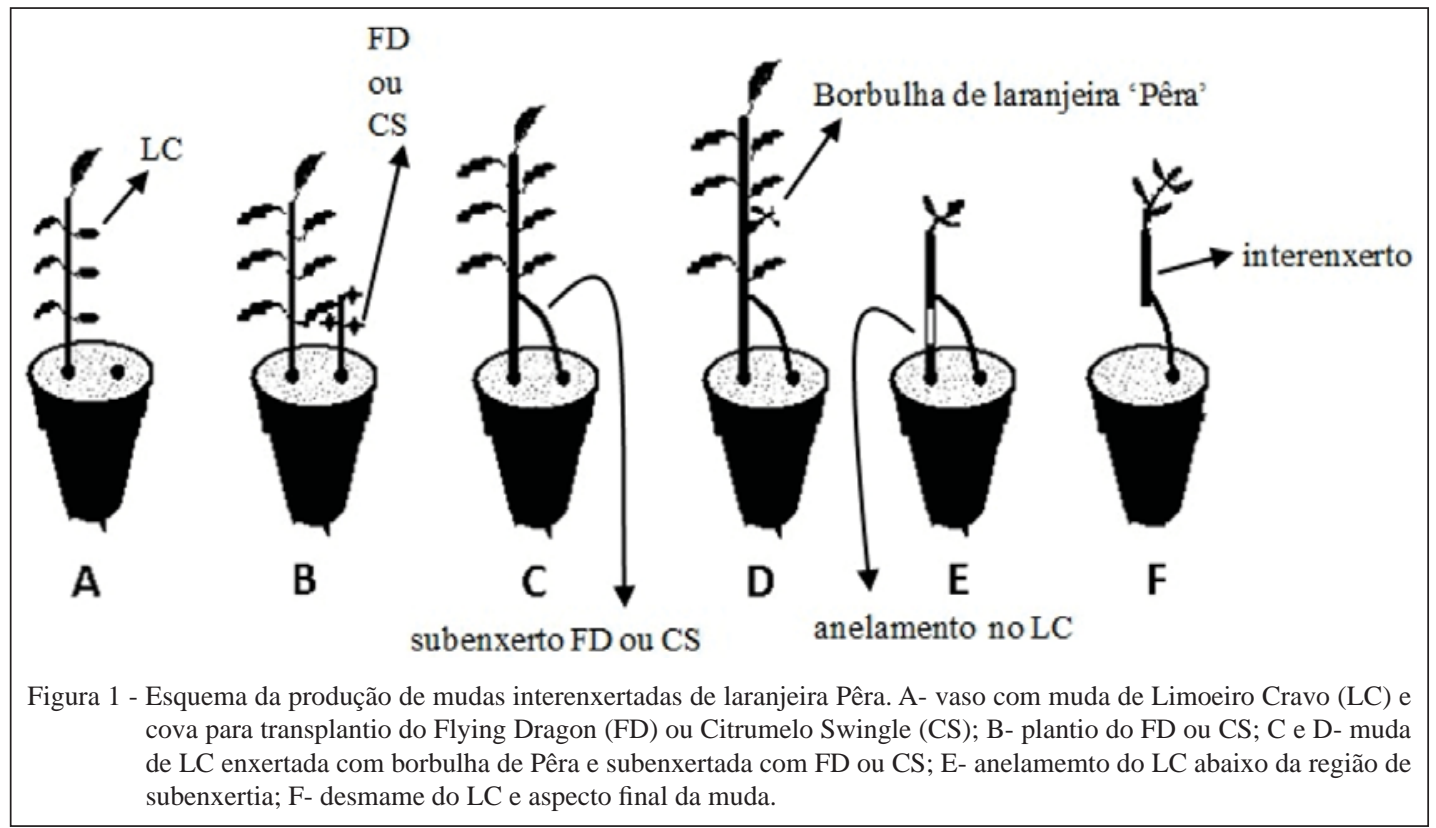

Foram avaliados 0 percentual de viabilidade das borbulhas enxertadas e os diâmetros dos interenxertos a $5 \mathrm{~cm}$ acima da região de enxertia. Os dados em parcela subdividida no tempo foram submetidos à análises de variância e as médias dos tratamentos, comparadas pelo teste Tukey $(\mathrm{P}=0,05)$.

Aos 30 DAE, foi observada maior porcentagem de viabilidade das borbulhas de laranjeira Pêra enxertadas no limoeiro Cravo (tratamentos 3, 4 e 5). Entretanto, nesta mesma época, as borbulhas de laranjeira Bahia enxertadas nos porta-enxertos FD e CS (tratamentos 1 e 2), tiveram baixo percentual de viabilidade das borbulhas (Tabela 2). Aos 60 DAE, observou-se redução no percentual de borbulhas vivas. Essa redução foi maior nos tratamentos 4 e 5, nos quais se realizou o anelamento no caule do LC na região abaixo da subenxertia (com FD ou CS). O baixo percentual de viabilidade das borbulhas pode ter ocorrido em função da falta de conexão dos tecidos condutores entre os subenxertos (FD e CS) e o filtro de LC. A má conexão entre os vasos condutores pôde ser observada posteriormente ao anelamento, com a murcha e seca dos brotos de laranjeira Pera e morte dos tecidos na região de subenxertia, sendo

Tabela 2 - Valores médios da porcentagem de viabilidade (PV) das borbulhas de laranjeira Bahia e Pêra e diâmetro do caule do interenxerto ou porta-enxerto (DCI) de mudas de laranjeira Pêra interenxertadas com laranjeira Bahia (Tratamentos 1 e 2 ), limoeiro Cravo (Tratamentos 4 e 5) e enxertadas diretamente em limoeiro Cravo (Tratamento 3).

\begin{tabular}{|c|c|c|c|c|c|c|}
\hline \multirow{3}{*}{ Tratamento } & \multirow{3}{*}{ Interenxerto } & \multicolumn{5}{|c|}{ 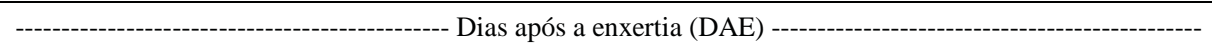 } \\
\hline & & 30 & 60 & 90 & 120 & 15 \\
\hline & & \multicolumn{2}{|c|}{ 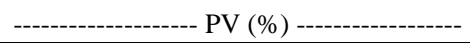 } & \multicolumn{3}{|c|}{---------------------------------DCI (mm)--------------------------- } \\
\hline 1 & Bahia & $30,00 \mathrm{c}$ & $18,75 \mathrm{c}$ & 2,53 a & 2,88 & 3,16 \\
\hline 2 & Bahia & $67,50 \mathrm{~b}$ & $42,5 \mathrm{~b}$ & 1,89 & 2,47 & 2,77 \\
\hline 3 & - & 96,43 a & 89,28 a & 8,00 & 8,48 & 8,64 \\
\hline 4 & Cravo & $100,00 \mathrm{a}$ & $25,00 \mathrm{c}$ & 7,63 & 8,04 & 8,22 \\
\hline 5 & Cravo & 89,23 a & $3,57 \mathrm{~d}$ & - & - & - \\
\hline média & & 76,64 & 35,82 & $5,01 \mathrm{C}$ & 5,47 B & $5,70 \mathrm{~A}$ \\
\hline CV (A) & & & 17,39 & & 6,68 & \\
\hline CV (B) & & & 11,67 & & 3,52 & \\
\hline
\end{tabular}

Nos tratamentos 1 e 2, foram enxertadas borbulhas de laranjeira Bahia e, nos 3, 4 e 5, foram enxertadas borbulhas de laranjeira Pêra. Nos tratamentos 1 e 2, os interenxertos foram formados com tecidos da laranjeira Bahia, enquanto, no tratamento 4 e 5, o interenxerto foi constituído por tecidos do limoeiro Cravo.

Médias seguidas de mesma letra minúscula na coluna e maiúscula na linha não diferem pelo teste tukey

Ciência Rural, v.44, n.3, mar, 2014. 
mais pronunciada no tratamento 5 , que envolveu a combinação Pêra / LC / CS.

A produção de mudas de laranjeira Pêra interenxertadas pelo sistema tradicional, tendo como porta-enxerto o CS ou o FD e o interenxerto de laranjeira Bahia, não foi possível, em virtude do baixo percentual de viabilidade e baixo vigor das brotações, que impediu nova enxertia. Esste atraso na formação do interenxerto acarretaria em maior atraso no tempo de produção da muda.

A combinação entre Pêra / LC / FD (T4) teve o melhor crescimento em diâmetro do interenxerto, chegando bem próximo das mudas produzidas sem interenxerto (Pêra / LC), indicando que a utilização do interenxerto LC pode ser uma alternativa para produção de mudas de laranjeira Pêra sobre trifoliatas. GIRARDI \& MOURÃO FILHO (2006) atribuem ao uso do interenxerto um atraso na produção de mudas da laranjeira de até 5 meses, em comparação com a muda produzida sem interenxerto, com aumento de descarte delas.

A subenxertia tem sido usada na citricultura brasileira em casos de necessidade da substituição de porta-enxertos, principalmente visando ao controle da morte súbita (SETIN et al., 2009). No caso deste trabalho, propõe-se maior avaliação do uso dessa técnica para introduzir um porta-enxerto abaixo do limoeiro Cravo, fazendo com que os tecidos desta variedade passem a funcionar como um interenxerto. O uso do LC como filtro ainda não foi avaliado no campo. Entretanto, a metodologia proposta poderá ser avaliada, também, para produção de mudas com interenxerto de outras variedades. Neste trabalho, foram produzidas mudas de laranjeira Pêra com interenxerto de LC e com o porta-enxerto FD. A avaliação dessa combinação em campo é de interesse, uma vez que o FD é um porta-enxerto nanicante, o que vai de encontro da nova necessidade da citricultura em aumentar a densidade de plantio.

\section{REFERÊNCIAS}

DONADIO, L.C. Laranja Pêra. Jaboticabal: Funep, 1999. 61p. (Boletim Citrícola 11).

FACHINELLO, J.C. et al. Propagação de plantas frutíferas. Brasilia: Embrapa Informação Tecnológica, 2005. 221p.

GIRARDI, E.A.; MOURAO FILHO, F. de A. A. Production of interstocked Pera sweet orange nursey trees on Volkamer lemon and Swingle citrumelo rootstocks. Scientia Agrícola, v.63, n.1, p.5-10, 2006. Disponível em: <http://dx.doi.org/10.1590/S010390162006000100002>. Acesso em: 01 jun. 2013. doi: 10.1590/ S0103-90162006000100002.

POMPEU JUNIOR, J.; BLUMER, S. Morte súbita dos citros: suscetibilidade de seleções de limão-cravo e uso de interenxertos. Revista Brasileira de Fruticultura, v.30, n.4, p.1159-1161, 2008. Disponível em: <http://dx.doi.org/10.1590/S010029452008000400052>. Acesso em: 01 jun. 2013. doi: 10.1590/ S0100-29452008000400052.

SETIN, D.W.et al. Crescimento inicial e estado nutricional da laranjeira Valência sobre porta-enxertos múltiplos de limoeiro Cravo e Citrumeleiro Swingle. Bragantia, v.68, n.2, p.397406, 2009. Disponível em: <http://dx.doi.org/10.1590/S000687052009000200014>. Acesso em: 01 jun. 2013. doi: 10.1590/ S0006-87052009000200014. 